Cite as: Auvray, M. (2019). Multisensory and spatial processes in sensory substitution. Restaurative Neurology and Neuroscience, 37, 609-619. doi: 10.3233/RNN-190950

\title{
Multisensory and spatial processes in sensory substitution
}

\section{Malika AUVRAY}

Institut des Systèmes Intelligents et de Robotique, CNRS UMR 7222, Université Pierre et Marie Curie, Paris, France

Address. ISIR, 4 Place Jussieu, 75005, Paris

Mail. auvray@isir.upmc.fr

\begin{abstract}
Sensory substitution devices aim at compensating sensory deficits by converting stimuli coming from a deficient sensory modality (e.g., vision) into stimuli accessible through another modality (e.g., touch or audition). Studies conducted with these devices revealed the central nervous system to be very plastic. Various laboratories have conducted studies investigating such plasticity by means of behavioural and brain-imaging techniques. At the ISIR Laboratory, we focused on the factors underlying the learning of sensory substitution devices, their adequacy to the target population, and we explored ways of improving their design by the use of crossmodal correspondences and by taking into account individual differences in the used reference frames. We also investigated the nature of the experience with sensory substitution. In particular, we suggested moving beyond positions reducing experience to that of a single sensory modality. Rather, sensory substitution is considered as a multisensory experience, involving not only visual, but also auditory or tactile processes as well as cognitive processes. In this framework, individual differences do have an influence on the extent to which the different sensory modalities influence the experience with the devices.
\end{abstract}

Keywords. Sensory substitution, Blindness, Brain plasticity, Rehabilitation technologies, Learning 


\section{Introduction}

Sensory substitution devices were developed with the aim of compensating one or several functions of a deficient sensory modality by converting stimuli that are normally accessed through this deficient sensory modality into stimuli accessible by another sensory modality. For instance, these devices can convert visual information into sounds or tactile stimuli. Sensory substitution devices have broad applications such as sensory rehabilitation and perceptual augmentation. For instance, visual-to-tactile (e.g., Bach-y-Rita et al., 1969; White et al., 1970) and visual-to-auditory (e.g., Hanneton et al., 2010; Meijer, 1992) conversion systems were designed in order to assist blind and visually impaired individuals. Numerous devices were designed to compensate other deficits, such as proprioceptive deficits (e.g. see Danilov et al., 2007; Diot et al., 2014). In the field of perceptual augmentation, these devices were developed in order to gain access to visual information, either when there is an excess of visual information to process, such as when driving a car (e.g., Ho and Spence, 2008) or when conditions of perception are degraded, as can be the case for those involved in firefighting (Carton and Dunne, 2013) or military operations (Jones et al., 2009).

In what follows, the focus will be on visual-to-tactile and visual-to-auditory devices, designed for the rehabilitation of visual deficits. Most of them consist of a tiny camera, either hand-held or embedded in glasses, recording the external scene in real time. This scene is then converted into sounds or tactile stimuli. The translation codes can be very diverse. In most visual-to-tactile devices, the translation code is analogical. For instance, a visual circle can be translated into a circular pattern of tactile stimuli. In some devices, non-analogical codes have also been used, for instance in order to convert distance into vibrations (e.g., Farcy et al., 2006; Maidenbaum et al., 2014, O’Brien, Auvray, Hayward, 2015). The code used in visualto-auditory devices translates several dimensions of the visual signal into dimensions of the auditory signal. For instance, the vOICe (Meijer, 1992) translates vertical position into frequency, horizontal position into time scanning, and visual brightness into auditory loudness. Other visual-to-auditory devices convert colour information using different orchestral instruments (e.g., the See ColOr; Bologna et al., 2009; the EyeMusic, Levy-Tzedek et al., 2014).

Studies conducted on sensory substitution devices revealed the central nervous system to be very plastic. Behavioural studies have investigated performance across a variety of tasks. In particular, studies have revealed that these devices allow their users to perform localisation tasks (e.g., Levy-Tzedek et al., 2012; Proulx et al., 2008), shape recognition (e.g., Arno et al., 2001; Auvray et al., 2007-a, 2007-b; Pollok et al., 2005; Striem-Amit et al., 2012b), and navigation tasks (Chebat et al., 2011; 2015). Brain-imaging studies have shown that the use and practice of visual-to-tactile (Ptito et al., 2005; see also Kupers et al., 2006) and visual-to-auditory (e.g., Striem-Amit and Amedi, 2014) devices result in increased activation in blind people's visual cortex (for reviews, see Proulx et al., 2014; Ptito et al., 2018). It should however be mentioned that one study directly compared activation longitudinally (Kim 
and Zatorre, 2011). The results did not reveal increased activation, but rather a change in functional connectivity. This result might suggest that the observed cross-modal recruitment of the visual cortex during the use of sensory substitution devices can be due to unmasking or existing computations through non-visual inputs already there prior to devices' use (e.g. Ptito et al., 2005; Striem-Amit et al., 2012-c).

Interestingly, the visual brain areas activated during a task with a visual-to-auditory device correspond to those that are activated for the same task performed visually. For instance, when congenitally blind participants are required to recognize human silhouettes with the visual-to-auditory conversion device The vOICe, this activates visual cortex extrastriate body-selective area, which responds more strongly to images of human body than to other types of objects (Striem-Amit and Amedi, 2014). Another example is the activation of visual word form area (VWFA) when congenitally blind participants are asked to recognize letters with The vOICe (Striem-Amit et al., 2012-b). This led the researchers in this group to think of the brain as having a flexible sensory-independent, task-selective supramodal organization rather than a sensory-specific one (Amedi et al., 2017; Heimler, Striem-Amit, \& Amedi, 2015).

Ever since their inception in the sixties, sensory substitution devices proved to be fruitful tools to investigate questions in the fields of philosophy of perception, experimental psychology, and studies of brain plasticity. However, in spite of their potential and the perspectives they open, sensory substitution devices remain under-used to date. Thus, one of the major challenges for scientists working in the field of rehabilitation is to favour the use of aid devices by impaired people. The limited success of sensory substitution devices can be explained by insufficiencies in the analyses of what is sensory substitution, insufficiencies in the understanding of impaired people's perceptual specificities, and deficiencies in the design of the devices. To overcome these insufficiencies, the research conducted in the lab lies both upstream and downstream of the design of sensory substitution devices. In particular, as will be detailed in the next sections, it involves a conceptual understanding of the nature and possibilities offered by sensory substitution devices, studies of the factors underlying learning processes, and the possibility of improving their design by using crossmodal correspondences and taking into account individual differences in the used reference frames.

\section{Conceptual understanding of sensory substitution}

One important question raised is: to which sensory modality does the perception with a sensory substitution device belong? Two opposite theses were initially put forward: the dominance thesis (e.g., Keeley, 2002) according to which perception with a sensory substitution device remains in the substituting modality (touch or audition), and the deference thesis (e.g., Hurley \& Noë, 2003; Noë, 2004; O’Regan, 2011) according to which perception switches to the substituted modality (vision). The deference thesis has opened the door to over-optimistic claims involving the idea that users of visual-to-tactile substitution devices 
would become able to "see with the skin" (White et al., 1970) or to "see with the brain" (Bach-y-Rita et al., 2003). These optimistic claims have been echoed to the wider audience, including potential users of these devices: sensory substitution devices have been advertised as "helping the blind see with their ears" (Jacobson, 2014 ) or "rewiring brains to see with sound" (Trivedi, 2010). My collaborators and I suggested that it is time to go beyond the dominance versus deference debate, and that perception with a sensory substitution device goes beyond assimilation to either the substituting or the substituted modality. According to this view, sensory substitution should be understood as being vertically integrated on preexisting capacities that involve the substituting and the substituted sensory modalities, as well as cognitive processes (see figure 1).

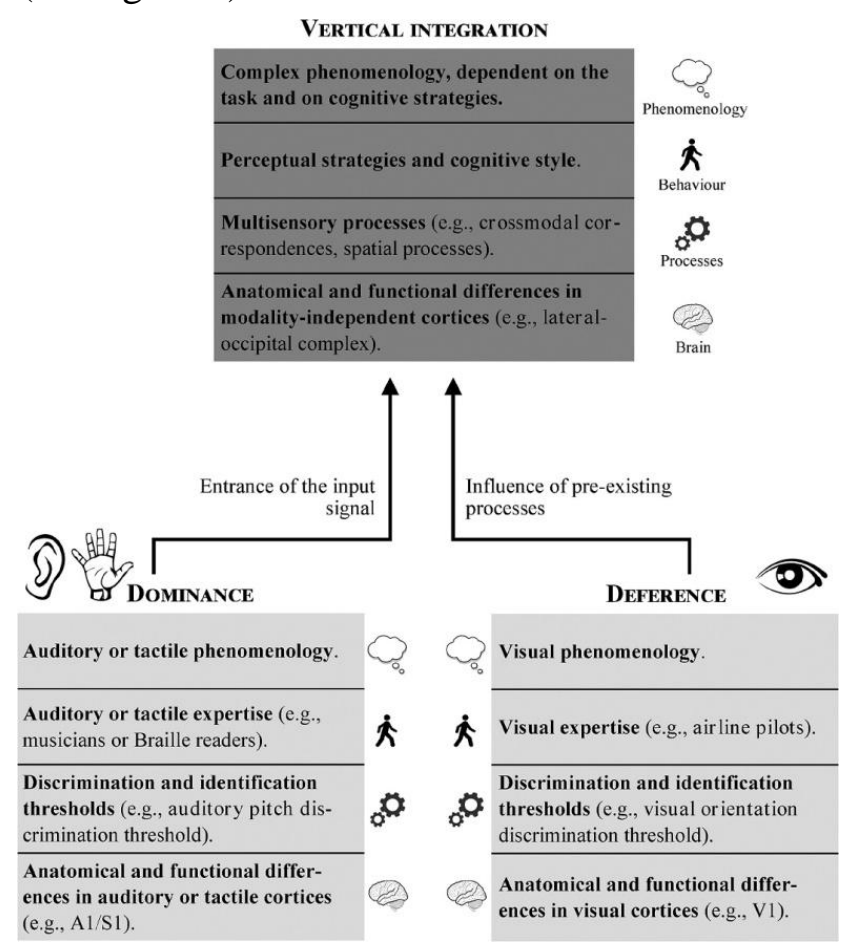

Figure 1 (figure from Arnold, Pesnot-Lerousseau, \& Auvray, 2017). Three alternative views on the processes involved in sensory substitution, with their specificities at the behavioural, neural, and phenomenological levels. Illustrative examples are given into brackets.

To go deeper into the defended view, the dominance versus deference debate remains based on an implicit perceptual equivalence which we suggest to identify as a perceptual assumption. The influence of this perceptual assumption is visible in the fact that researchers accept or target equivalences between using a sensory substitution device and the exercise of a sensory modality. In other words, the perceptual assumption considers that sensory substitution follows what occurs with canonical cases of perception through one of the typical sensory modalities that is as specialized channels for transducing external information. To us, this perceptual assumption has led to a confirmation bias in the interpretation of the results. The results of existing studies have been filtered out of the negative evidence, or data fitting less well with this assumption, while the remaining evidence has been seen as confirming the 
equivalence between using a sensory substitution device and perceiving through one of the canonical senses. Furthermore, the experimental protocols themselves are built with the perceptual assumption in mind which, in turn, constrains or limits the kind of data that can be gathered. As will be detailed below, our aim was to stress the limits of the perceptual models and the perspectives that can be opened by alternative models.

We first discussed the dominance versus deference debate by having recourse to the criteria that have been used for the taxonomy of our sensory modalities (Auvray \& Myin, 2009). These criteria involve sensory organ, stimuli, properties, qualitative experience, behavioural equivalence, dedication, and sensorimotor equivalence. The reviewed criteria did not allow favouring the dominance or the deference theses, and most of them appeared as a matter of degree or a matter of interpretation. We subsequently argued that the evidence leads to an alternative view, according to which the experience after sensory substitution is a transformation, extension, or augmentation of our capacities rather than being equivalent or reducible to an already existing sensory modality. We developed this view by comparing sensory substitution devices to other "mind enhancing tools" such as pen and paper, sketchpads or calculators. In particular, we built on the transformative view of mind enhancing tools (e.g., Clark, 2003; Menary, 2006) according to which they do transform cognition in a qualitative way. In this sense, these tools not only facilitate established cognitive processes, they can also allow for the appearance of novel cognitive operations, which simply would have been impossible without them.

This view was subsequently refined to include the cognitive processes at stake (see Deroy \& Auvray, 2012, 2014). In particular, we argued that learning to use a sensory substitution device should not be thought of as occurring horizontally. Experience with sensory substitution devices is built up from existing sensory modalities and this relation is of interdependence or crafting. We used an analogy with dual-route models of reading (e.g., Coltheart \& Rastle, 1994), according to which, learning to use a sensory substitution device is no longer to be thought of as being merely a matter of perceptual learning or adaptation, but as the building of a parallel access to cognitive and spatial representations that get grafted onto some pre-existing perceptual-cognitive route. To summarize, sensory substitution is treated here as involving both perceptual and cognitive processes, which take into account integration of the novel information with the existing perceptual-semantic route.

While developing this view, it appeared necessary to take into consideration alternative views on sensory substitution and in particular the claims that long-term use of sensory substitution devices may induce forms of synaesthesia in practised users (e.g., Proulx, 2010; Ward \& Meijer, 2010; Ward \& Wright, 2014). We proposed to clarify the debate by dissociating the substituted information from the associated phenomenology and to verify for each, if they fulfil the criteria used to define congenital synaesthesia (i.e., inducer-concurrent pairing, idiosyncrasy, automaticity of the process, consistency over time). Our analysis highlighted that comparing the two is not straightforward. Indeed, defending SSD-use as 
being synesthetic involves giving an account of why one or several criteria to define synesthesia can be loosened in some cases without weakening the understanding and definitions given to congenital synesthesia. In addition, our theoretical analyses suggest that whatever the criteria, experience with a sensory substitution device appears to be closer to cross-modal correspondences than to synaesthesia (Auvray \& Farina, 2017).

Finally, we built on the vertical integration model in order to investigate the predictions that can be made from this model (Arnold, Pesnot-Lerousseau, \& Auvray, 2017). In particular, the vertical integration model involves the idea that, at the perceptual level, performance and experience with the device will depend on each person's abilities. Secondly, if the use of sensory substitution devices is both perceptual and cognitive, then this leaves room for perceptual strategies that will differ depending on people's specificities and cognitive style. In other words, performance and experience with the device will then be done with different weights attributed to each of the sensory modalities. These weights will depend on people's individual capacities, perceptual preferences, and cognitive styles. Note that these weights might subsequently vary depending on expertise, mastery of the device, and type of task. For example, a musician might be able to perform auditory analyses that non-musicians would not, consequently giving more weight to auditory processes, and possibly having a predominant auditory experience when using the device.

In order to start and behaviourally investigate the vertical integration hypothesis, we conducted an experiment with the visual-to-auditory conversion device The vOICe (Meijer, 1992), which translates visual images into soundscapes. Before and after training, participants were tested to see whether auditory stimuli would spontaneously evoke visual images (Pesnot-Lerousseau, Arnold, \& Auvray, personal communication). This stoop-like paradigm (Stroop, 1935) consisted in a sound recognition task combined with a simultaneous presentation of visual distractors. The sounds corresponded to the auditory conversion of visual lines with The vOICe. Visual distractors, presented simultaneously, could have either the same orientation as the sound or a different one. In addition, we measured participants' auditory abilities and performance with the device to see if they correlate. Our results revealed a stroop-like effect only after training, suggesting that people visualize auditory stimuli. In addition, participants' performance during training for localisation and recognition tasks as well as their associated phenomenologies depended on their auditory abilities.

Overall, these results suggest that the plasticity at stake in sensory substitution is complex, and based on a multisensory architecture involving both visual and auditory processes. They are in line with the vertical integration thesis model (Arnold et al., 2017). These results are also in line with the complementary metamodal/ supramodal view of sensory substitution processing (Cecchetti et al., 2016; Heimler et al., 2015; Proulx et al., 2014, 2016) which proposes that the functional plasticity at stake occurs at higher levels, involving supramodal representations of shapes with a spatial integration of visual and auditory stimuli. More generally, these results highlight that sensory plasticity in humans is a complex 
phenomenon which depends both on the kind of processes that are involved and on individual specificities. Several questions remain open and are currently under scientific investigation in our lab, in particular the question of whether the interference appears at the visual or at a supramodal level, as well as the influence of visual imagery in the processes.

\section{Sensory substitution and learning}

An important feature in mastering sensory substitution devices is how easy they are to use and how much training they require on the users' part. For instance, with the TVSS, extensive training is required. Most users are able to explore their environment, approximate objects' positions, and describe their raw shape within a couple of hours. The training period required to reach a more complete level of performance is estimated at around 8 hours with visual-to-tactile devices (Kaczmarek \& Haase, 2003) and 10 to 15 with visual-to-auditory devices (Auvray et al., 2007-a), and an even a longer time to perform fine-grained tasks, such as recognizing body postures (Striem-Amit \& Amedi, 2014) with visual-to-auditory devices. It is thus crucial to investigate users' learning abilities, to identify the most efficient learning methods and those that are adapted to different users' profile. Another crucial characteristic of learning lies in the ability to generalize, that is, the ability to extend the acquired perceptual abilities to new bodily locations, new stimuli, and new perceptual conditions.

Regarding bodily locations, one important claim of Bach-y-Rita is that, once trained, users of visual-to-tactile sensory substitution devices no longer feel the stimulation on their skin, where it occurs, but they directly attribute the stimulation as resulting from an external object, i.e. located at a distance (Bach-y-Rita \& Kercel, 2003). Bach-y-Rita then asserted that, consequent to this externalization process, the tactile stimulator array can be moved from one body surface to another, without loss in spatial localization abilities or other perceptual capacity. This claim, although recurrent, was only based on few users' verbal reports. In order to investigate it with objective methods, participants in our study were trained to recognize tactile letters on one body part (belly, thigh, or shin) and their performance were tested preand post-training on all three body surfaces. Our results revealed that participants' performance (both in terms of accuracy and reaction times) improved, and this improvement was similar for surfaces represented in adjacent (e.g., belly and thigh) and in non-adjacent (e.g., belly and shin) areas of the somatosensory cortex (Arnold, \& Auvray, 2014).

This result provides behavioural support for the claim that training in sensory substitution results in a relative independence from the stimulated body surface. These results have implications for the use of sensory substitution devices and vibrotactile systems by visually impaired people. First, they suggest that training on one body surface is beneficial to the entire body. As a consequence, users do not need to undergo an extensive training on all body surfaces. This study also provides better understanding of the learning process, which is a crucial feature for efficient use of tactile devices. It reveals that transfer of learning to other body surfaces occurs within a relatively short time frame (i.e., less than $90 \mathrm{~min}$ ). 
In a subsequent study, we investigated the extent to which learning transfers to novel perceptual conditions and to novel stimuli (Arnold, \& Auvray, 2018). The learning protocol consisted in alternating repeated and new lists of tactile alphanumerical stimuli. Our results showed a transfer of learning to novel orientations. In addition, they showed that the ability to generalize to new symbols depend on the number of stimuli in the list and on feature variability. Our results are in line with the reverse hierarchy theory (RHT) of perceptual learning (Ahissar \& Hochstein, 2004). According to this theory, the degree of specificity depends on the difficulty of the perceptual task and on the level of cortical processing that is required to perform the task. For a difficult task involving low-level cortical processes, perceptual learning is specific to the characteristics of the task and it does not generalize to new characteristics. On the other hand, for an easier task, involving higher-level processing, perceptual learning is less specific to the low-level characteristics of the task or of the stimuli. This study has applied implications for the design of learning protocols for sensory substitution. In particular, the results suggest that short training sessions with high variability and diversity will facilitate generalization and will allow for a more optimal use of the device in real-life conditions. It should be added that beyond the nature and variability of the stimuli sample, the instructions given to users during training also has an influence on generalization abilities. For instance, in a study by Striem-Amit et al. (2012-a), the participants were requested to describe the entire detailed shape of the image provided by the visual-to-auditory conversion device The vOICe before identifying it. The results showed wide generalization in this condition, in line with reverse hierarchy theory of perceptual learning.

Finally, during learning, there are important individual differences to take into account. For instance, it was found that people with good auditory abilities (Pesnot-Lerousseau et al., personal communication), good musical abilities (Haigh et al., 2013), or high visuo-spatial abilities (Hanneton et al., 2015; although see Brown et al., 2011) have better performance when using visual-to-auditory substitution devices. Differences also occur as a function of visual experience. In particular, regarding navigation tasks, two reference frames are used: an egocentric reference frame, based on body coordinates and characterized by a serial aspect, and an allocentric reference frame based on cardinal coordinates. The allocentric reference frame is characterized by a high level of flexibility and it allows reorganization of itineraries such as taking shortcuts or finding alternative routes. The construction of an allocentric reference frame is facilitated by vision as this sensory modality gives access to a simultaneous multiple perception of distant spatial elements. Consequently, sighted, early-blind, and late blind people differ in the adopted reference frame (e.g., see Noordzij et al., 2006; Pasqualotto et al., 2013, Rieser et al., 1986). In general, sighted and late-blind participants prefer to adopt an allocentric reference frame, whereas congenitally blind participants prefer to adopt an egocentric one (see Gallay et al., 2013; Pasqualotto et al., 2012; Schinazi et al., 2016, for reviews). Differences also occur in the way people plan and perform an itinerary, for which blind and sighted people use different heuristics and procedures (Brunet et al., 2018). In 
addition, blind participants have been shown to be better than sighted participants at obstacle avoidance when using a sensory substitution device (Kolarik et al., 2016). These results suggest that performance with the device relies on users' individual sensory abilities and that learning protocols will benefit from taking them into account.

\section{Improving the design of sensory substitution devices}

One crucial question when designing sensory substitution devices is how best to code visual dimensions into tactile or auditory dimensions. Each category of devices raises questions and in particular, visual-to-auditory conversion devices raise the issue of matching the sensory dimensions of the signal and visual-to-tactile conversion devices raise the question of the reference frame according to which information has to be encoded. Thus, with visual-to-auditory devices, several dimensions of the visual information (most of the time horizontal and vertical dimensions, depth, brightness, and sometimes colour) are coded using several auditory dimensions (most of the time pitch, time scanning, inter-aural differences, loudness, and timber). The question arises as to how to determine the pairings between auditory and visual dimensions. The four main visual-to-auditory devices (e.g., the vOICe, Meijer, 1992; The PSVA, Capelle et al., 1998; Vibe, Hanneton, Auvray, \& Durette, 2010; the EyeMusic, Abboud et al., 2014) have been designed by using the correspondence between direction of pitch and direction of movement (higher-higher; lower-lower). However, no one tested whether this correspondence, which was intuitive for the designers and welldocumented in audiovisual contexts for sighted individuals (see Parise \& Spence, 2012; Spence, 2011), was also relevant for blind people.

In order to investigate the relevance of the pitch-elevation correspondence for blind persons, we tested the audio-tactile analogue of this correspondence (Deroy, Fasiello, Hayward, \& Auvray, 2016). An implicit association test was used, in which auditory and tactile stimuli were presented sequentially and associated to different response buttons. Our results crucially showed that the pitch-elevation correspondence exists in sighted people but is absent in both early and late blind people. This results calls for a rethinking of the codes used in sensory substitution and one of our research axes is to investigate more systematically the possible crossmodal correspondences between audition, vision, and touch focusing on the dimensions of the signals that are relevant for sensory substitution. We also investigate the ways in which these correspondences are combined together, as they are, so far, only investigated in isolation.

With visual-to-tactile devices, it is important to take into account the reference frame in which the information is encoded. Indeed, tactile stimulation received on the body surface can be interpreted from a body location (i.e. self-centred perspective) or from an external location (i.e. decentred perspective, e.g., see Sekiyama, 1991, Arnold, Spence, \& Auvray, 2017, for a review). These different perspectives can correspond to a mental localization of the tactile stimulation either on the body or on the external space (see figure 2). In order to investigate 
spatial reference frames, we used a graphesthesia task, consisting of recognizing ambiguous symbols (the letters b, d, p, and q) drawn on the body surface by means of a tactile matrix. The participants' response allows determining the adopted perspective. Take the example of the lowercase letter "b" (from an external perspective) drawn on the participants' stomach. Recognizing the letter " $b$ " requires the participant to take a decentred perspective (i.e., decentred from the participant's position and centred on that of the experimenter). Recognizing letter "d" requires taking a trunk-self-centred perspective, as if the letter were to be projected forward and "seen" from the participant's position. Recognizing the letter "q" requires taking a head-self-centred perspective, as if the head was bending forward to "see" the letter "q" on the stomach. In addition to investigating the perspectives that are naturally adopted, the graphesthesia task, by alternating imposed self-centred and decentred reference frames, allows investigating the flexibility in changing perspectives.

A

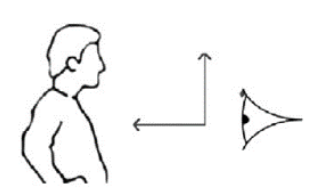

Decentred perspective

(facing toward)

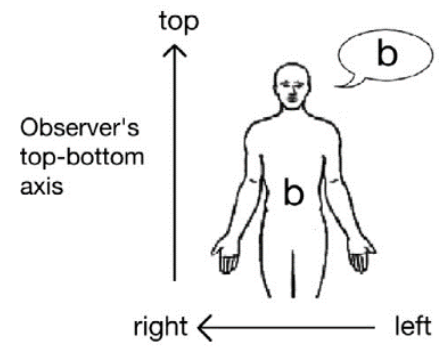

Observer's left-right axis
B

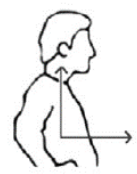

Trunk-centred perspective (facing forward)

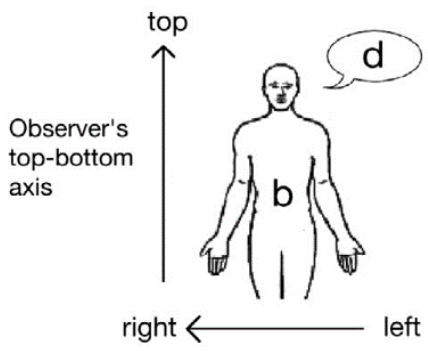

Observer's left-right axis

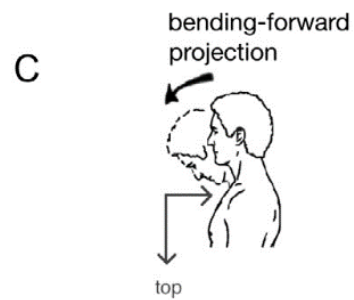

Head-centred perspective

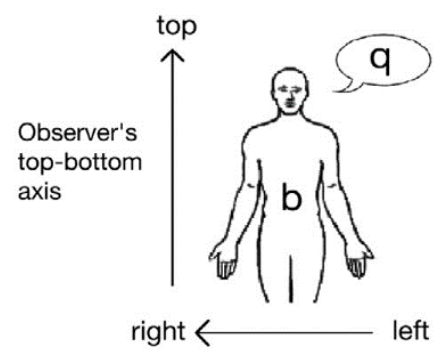

Observer's left-right axis

Figure 2 (figure from Arnold, Spence, \& Auvray, 2017). Illustration of the different spatial perspectives that can be adopted in the graphesthesia task, with the example of the tactile letter " $b$ " drawn by the experimenter.

Firstly, our studies showed that there is an important individual variability, with $50 \%$ of the sighted participants preferring to perceive space from a trunk-centred perspective, $29 \%$ from a head-centred perspective and $21 \%$ from a decentred perspective (Arnold, Spence, \& Auvray, 2017). This reference frame appeared to be natural and cannot be reduced to a mere cognitive choice (Arnold, Spence, \& Auvray, 2016). Secondly, our studies revealed the influence of vision on the reference frames that were adopted with i) blind people adopting less frequently the decentred perspectives; ii) the (temporary or permanent) lack of vision favouring the head-self-centred perspective, and iii) early-blind people having more difficulties than sighted in switching reference frames (Job et al., personal communication). 
Additional studies allowed showing variability as a function of where the stimuli were located on the body surface: more specifically, the head-centred perspective is easier to adopt for surfaces that can be easily looked at with real head movements (Arnold \& Auvray, 2017). Similarly, the adopted perspective varied for stimuli presented on the hand, with differences as a function of the orientation of the hand and differences between hand and palm (O'Brien \& Auvray, 2016). This suggests that the codings for visual-to-tactile sensory substitution devices might benefit from taking into account the location of the tactile matrix as well as inter-individual differences. We are currently using this paradigm to investigate the mechanisms of distal attribution with visual-to-tactile sensory substitution devices (see O'Brien \& Auvray, 2014, for a review on distal attribution). In particular, our aim is to investigate how, with the help of training, users might transition from sensing the tactile stimuli on their body to perceiving the object as being located in front of them, in the 3-D space.

Questions are frequently raised concerning the location of the camera used to explore the scene. For instance, according to sensorimotor theories of perception, the use of a headmounted camera shares more sensorimotor resemblance with vision than the use of a handheld camera and thereby might favour better performance (e.g., O'Regan, 2011). We investigated participants' performance in a localization task with a visual-to-auditory device when the target's position was computed either from the participants' hand of from their head (Hanneton et al., 2019). Our results revealed that performance in hand mode was similar to that in head mode, and that improvements were slower in head mode. Additional analyses revealed that performance was less linked to the natural aspect related to sensor location than to the spatial coincidence between the sensor and the body part performing the motor task and to the latter's degrees of freedom. It should be added that different results might be obtained as a function of visual experience. Indeed, sighted and late blind persons might find it easier to explore a scene with the sensors located on the head than early blind persons.

Finally, in sensory substitution, two main trends are possible: either developing generalist devices aiming at compensating for a maximum of lost visual functions, or, conversely, targeting specific perceptual functions (localization, recognition, navigation) that can be impaired in blind people. One hypothesis we raised is that one of the reasons of the under-use of sensory substitution devices lies on the unrealistic promises of a generalist device that would restore the entirety of a deficient sensory modality. By contrast, blind persons more easily use technical devices devoted to a specific function (such as auditory or tactile navigation devices or software to navigate the internet, Deroy \& Auvray, 2012). This hypothesis remains to be experimentally tested and is the topic of future studies.

\section{General discussion}

Although sensory substitution seems a promising technology to compensate visual impairments, as of today, the developed devices are barely used by visually impaired 
individuals, either because the tools' design is not optimal, or because their use involves too many constraints and cognitive effort. At the applied level, the studies developed in our lab help in the design of sensory substitution devices. Firstly, the learning protocols will benefit from taking into account specific and generalized conditions of learning in order to favour the transfer of learning to novel stimuli and novel perceptual conditions. Secondly, taking into account individual differences, as suggested by the vertical integration hypothesis, will allow optimal appropriation of these devices. For example, we could identify different perceptual strategies associated to different phenomenologies, with some people having more of a visual use of the device and other people having more of an auditory or tactile use. Then the learning protocols could be customized as a function of the different types of use (see also Elli et al., 2014). For people having more auditory experiences, learning could be oriented toward favouring the auditory processes. This can be done by learning to better discriminate auditory intensities and frequencies. For people having more visual experiences, learning could favour tasks requiring visuo-spatial imagery, or tasks designed to better apprehend depth, to learn how to form global images of the objects. Third, the choice of the conversion codes for visual-to-auditory devices will benefit from the identification of the crossmodal correspondences existing in the different target populations. Finally, the design of the devices can be improved by taking into account the reference frames adopted by people (trunkcentred, head-centred, or decentred), for instance by implementing flexible options. Regarding the conceptual aspects, our studies help making advances in the understanding of the processes involved when using a sensory substitution device. Our results with visual-toauditory devices show that experience takes roots both on the visual and the auditory sensory modalities. Our results with tactile devices show that performance depends on both the task and individual abilities. This suggests that there is room for processes that are more cognitive, and thus more subject to individual variability. These results are in line with the vertical integration hypothesis, according to which sensory substitution devices are vertically integrated on pre-existing capacities that involve cognitive and perceptual processes.

\section{References}

Abboud, S., Hanassy, S., Levy-Tzedek, S., Maidenbaum, S., \& Amedi, A. (2014). EyeMusic: Introducing a "visual" colorful experience for the blind using auditory sensory substitution. Restorative Neurology and Neuroscience, 32, 247-257.

Ahissar, M., \& Hochstein, S. (2004). The reverse hierarchy theory of visual perceptual learning. Trends in Cognitive Science, 8, 457-464.

Amedi, A., Hofstetter, S., Maidenbaum, S., \& Heimler, B. (2017). Task selectivity as a comprehensive principle for brain organization. Trends in Cognitive Sciences, 21, 307-310.

Arno, P., De Volder, A. G., Vanlierde, A., Wanet-Defalque, M. C., Streel, E., Robert, A., et al. (2001). Occipital activation by pattern recognition in the early blind using auditory substitution for vision. Neuroimage, 13, 632-645.

Arnold, G., \& Auvray, M. (2018). Tactile recognition of visual stimuli: Specificity versus generalization of perceptual learning. Vision Research, 152, 40-50. 
Arnold, G., \& Auvray, M. (2014). Perceptual learning: Tactile letter recognition transfers across body surfaces. Multisensory Research, 27, 71-90.

Arnold, G. \& Auvray, M. (2017). The graphesthesia paradigm: drawing letters on the body to investigate the embodied nature of spatial perspective taking. I-Perception, 8, 1-5.

Arnold, G., Pesnot-Lerousseau, J. \& Auvray, M. (2017). Individual differences in sensory substitution. Multisensory Research, 6, 579-600.

Arnold, G., Spence, C., \& Auvray, M. (2016). Taking someone else's spatial perspective: Natural stance or effortful decentring? Cognition, 148, 27-33.

Arnold, G., Spence, C., \& Auvray, M. (2017). A unity of the self or a multiplicity of locations? How the graphesthesia task sheds light on the role of spatial perspectives in bodily self-consciousness. Consciousness \& Cognition, 56, 100-114.

Auvray, M., \& Farina, M. (2017). Patrolling the boundaries of synaethesia: A critical appraisal of transient and artificially-acquired forms of synaesthetic experiences. In $\mathrm{O}$. Deroy (Ed.). Synaesthesia: Philosophical \& Psychological Challenges (pp. 248-274), Oxford: Oxford University Press.

Auvray, M., Hanneton, S., \& O'Regan, J. K. (2007-a). Learning to perceive with a visuoauditory substitution system: Localization and object recognition with The Voice. Perception, 36, 416-430.

Auvray, M., \& Myin, E. (2009). Perception with compensatory devices. From sensory substitution to sensorimotor extension. Cognitive Science, 33, 1036-1058.

Auvray, M., Philipona, D., O'Regan, J. K., \& Spence, C. (2007-b). The perception of space and form recognition in a simulated environment: The case of minimalist sensorysubstitution devices. Perception, 36, 1736-1751.

Bach-y-Rita, P., Collins, C. C., Saunders, F. A., White, B., \& Scadden, L. (1969). Vision substitution by tactile image projection. Nature, 221, 963-964.

Bach-y-Rita, P., \& Kercel, S. W. (2003). Sensory substitution and the human-machine interface. Trends in Cognitive Sciences, 17, 541-546.

Bach-y-Rita, P., Tyler, M. E., \& Kaczmarek, K. A. (2003). Seeing with the brain. International Journal of Human-Computer Interaction, 2, 285-295.

Bologna, G., Deville, B., \& Pun, T. (2009). On the use of the auditory pathway to represent image scenes in real-time. Neurocomputing, 72, 839-849.

Brown, D. J., Macpherson, T., \&Ward, J. (2011). Seeing with sound? Exploring different characteristics of a visual-to-auditory sensory substitution device, Perception, 40, 11201135.

Brunet, L. Darses, F., \& Auvray, M. (2018). Strategies and needs of blind pedestrians during urban navigation. Le Travail Humain, 81, 141-171.

Capelle, C., Trullemans, C., Arno, P., \& Veraart, C. (1998). A real-time experimental prototype for enhancement of vision rehabilitation using auditory substitution. IEEE Transaction on Biomedical Engineering, 45, 1279-1293.

Carton, A., \& Dunne, L. E. (2013). Tactile distance feedback for firefighters: design and preliminary evaluation of a sensory augmentation glove. In Proceedings of the 4th Augmented Human International Conference (AH '13). ACM, New York, NY, USA, 5864.

Cecchetti, L., Kupers, R., Ptito, M., Pietrini, P., \& Ricciardi, E. (2016). Are supramodality and cross-modal plasticity the yin and yang of brain development? From blindness to rehabilitation. Frontiers in Systems Neuroscience, 10.

Chebat, D. R., Maidenbaum, S., \& Amedi A. (2015). Navigation using sensory substitution in real and virtual mazes. PLOS ONE, 10, e0126307.

Chebat, D. R., Schneider, F. C., Kupers, R., \& Ptito, M. (2011). Navigation with a sensory substitution device in congenitally blind individuals. Neuroreport, 22, 342-347. 
Clark, A. (2003). Natural-born cyborgs: Minds, technologies, and the future of human intelligence. New York: Oxford University Press.

Coltheart, M., \& Rastle, K. (1994). Serial processing in reading aloud: evidence for dual-route models of reading. Journal of Experimental Psychology Human Perception and Performance, 20, 1197-1211.

Danilov, Y., Tyler, M., Skinner, K., Hogle, R., \& Bach-y-Rita, P. (2007). Efficacy of electrotactile vestibular substitution in patients with peripheral and central vestibular loss, Journal of Vestibular Research, 17, 119-130.

Deroy, O., \& Auvray, M. (2012). Reading the world through sensory substitution devices. Frontiers in Theoretical and Philosophical Psychology, 3, 457.

Deroy, O., \& Auvray, M. (2014). A crossmodal perspective on sensory substitution. In S. Biggs, M. Matthen, \& D. Stokes (Eds.), Perception and Its Modalities (pp. 327-349), Oxford: Oxford University Press.

Deroy, O., Fasiello, I., Hayward, V., \& Auvray, M. (2016). Differentiated audio-tactile correspondences in sighted and blind individuals, Journal of Experimental Psychology: Human Perception and Performance, 42, 1204-1214.

Diot, B., Halavackova, P., Demongeot, J., \& Vuillerme, N. (2014). Sensory substitution for balance control using a vestibular-to-tactile device, Multisensory Research, 27, 313-336.

Elli, G. V., Benetti, S. and Collignon, O. (2014). Is there a future for sensory substitution outside academic laboratories? Multisensory Research, 27, 271-291.

Farcy, R., Leroux, R., Jucha, A., Damaschini, R., Grégoire, C., \& Zogaghi, A. (2006). Electronic travel aids and electronic orientation aids for blind people: Technical, rehabilitation and everyday life points of view. In M. A. Hersh (Ed.), Proceedings of the Conference \& Workshop on Assistive Technology for Vision and Hearing Impairment.

Gallay, M., Denis, M., \& Auvray, M. (2013). Navigation assistance for blind pedestrians: Guidelines for the design of devices and implications for spatial cognition. In T. Tenbrink, J. Wiener, \& C. Claramunt (Eds.), Representing space in cognition: Interrelations of behaviour, language, and formal models, Oxford: Oxford University Press.

Haigh, A., Brown, D. J., Meijer, P., \& Proulx, M. J. (2013). How well do you see what you hear? The acuity of visual-to-auditory sensory substitution, Frontiers in Psychology, 4, 330.

Hanneton, S., Auvray, M., \& Durette, B. (2010). The Vibe: A versatile vision-to-audition sensory substitution device. Applied Bionics and Biomechanics, 7, 269-276.

Hanneton, S., Herquel, P., \& Auvray, M. (2015). Intermodal recoding of a video game: Learning to process signals for motion perception in a pure auditory environment. International Journal of Adaptive Control and Signal Processing, 29, 1475-1483.

Hanneton, S., Hoellinger, T., Forma, V., Roby-Brami, A. \& Auvray, M. (2019/in press). Ears on the hand. Reaching three-dimensional targets with an audio-motor device. Multisensory Research.

Heimler, B., Striem-Amit, E., \& Amedi, A. (2015). Origins of task-specific sensoryindependent organization in the visual and auditory brain: neuroscience evidence, open questions and clinical implications. Current Opinion in Neurobiology, 35, 169-177

Ho, C. \& Spence, C. (2008) The multisensory driver: Implications for ergonomic car interface design. Aldershot: Ashgate Publishing.

Hurley, S., \& Noë, A. (2003). Neural plasticity and consciousness. Biology and Philosophy, $18,131-168$.

Jacobson, R. (2014). App helps the blind "see" with their ears. National Geographic, 5 April 2014.

Job, X., Arnold, G., Kirsh, L., \& Auvray, M. (personal communication). Blindness and temporary visual deprivation affects perspective-taking on tactile information 
Job, X., Kirsh, L., Inard, S., Arnold, G., \& Auvray, M. (in press). Spatial perspective taking is related to social intelligence and attachment style. Personality and Individual Differences.

Jones, L. A., Kunkel, J., \& Piateski, E. (2009). Vibrotactile pattern recognition on the arm and back. Perception, 38, 52-68.

Kaczmarek, K. A., \& Haase, S. J. (2003). Pattern identification and perceived stimulus quality as a function of stimulation current on a fingertip-scanned electrotactile display. IEEE Transaction on Neural System Rehabilitation Engineering, 11, 9-16.

Kim, J. K., \& Zatorre, R. J. (2011). Tactile-auditory shape learning engages the lateral occipital complex. Journal of Neuroscience, 31, 7848-7856.

Kolarik, A. J., Scarfe, A. C., Moore, B. C. J., \& Pardhan, S. (2017) Blindness enhances auditory obstacle circumvention: Assessing echolocation, sensory substitution, and visualbased navigation. PLoS ONE 12, e0175750.

Kupers, R., Fumal, A., de Noordhout, A. M., Gjedde, A., Schoenen, J., \& Ptito, M. (2006) Transcranial magnetic stimulation of the visual cortex induces somatotopically organized qualia in blind subjects. Proceedings of the National Academy of Science of the USA, 103, 13256-13260.

Levy-Tzedek, S., Novick, I., Arbel, R., Abboud, S., Maidenbaum, S., Vaadia, E., \& Amedi, A. (2012). Cross-sensory transfer of sensory-motor information: visuomotor learning affects performance on an audiomotor task, using sensory-substitution. Scientific Reports, $2,949$.

Levy-Tzedek, S., Riemer, D., \& Amedi, A. (2014). Color improves "visual" acuity via sound. Frontiers in Neuroscience, 8, 358.

Maidenbaum, S., Hanassy, S., Abboud, S., Buchs, G., Chebat, D. R., Levy-Tzedek, S., \& Amedi, A. (2014). The "EyeCane", a new electronic travel aid for the blind: Technology, behavior \& swift learning. Restorative Neurology and Neuroscience, 32, 813-824.

Meijer, P. B. L. (1992). An experimental system for auditory image representations. IEEE Transactions on Biomedical Engineering, 39, 112-121.

Noë, A. (2004). Action in Perception. Cambridge, MA: MIT Press.

Noordzij, M. L., Zuidhoek, S., \& Postma, A. (2006). The influence of visual experience on the ability to form spatial mental models based on route and survey descriptions, Cognition 100, 321-342.

O’Brien, J., \& Auvray, M. (2016). Cognition overrides orientation dependence in tactile viewpoint selection. Experimental Brain Research, 234, 1885-1892.

O'Brien, J., Auvray, M., \& Hayward, V. (2015). Perception of distance-to-obstacle through time-delayed tactile feedback. Proceedings of the 2015 IEEE World Haptics Conference (pp. 7-12). doi: 10.1109/WHC.2015.7177683

O'Brien, J., \& Auvray, M. (2014). The process of distal attribution illuminated through studies of sensory substitution. Multisensory Research, 27, 421-441.

O'Regan, J. K. (2011). Why Red Doesn't Sound Like a Bell. Understanding the Feel of Consciousness. Oxford: Oxford University Press.

Parise, C. V., \& Spence, C. (2012). Audiovisual crossmodal correspondences and sound symbolism: A study using the implicit association test. Experimental Brain Research, 220, 319-333.

Pasqualotto, A., \& Proulx, M. J. (2012). The role of visual experience for the neural basis of spatial cognition, Neurosciences and Biobehavoural Reviews, 36, 1179-1187.

Pasqualotto, A., Spiller, M. J., Jansari, A. S., \& Proulx, M. J. (2013). Visual experience facilitates allocentric spatial representation, Behavioural Brain Research, 236, 175-179.

Pesnot-Lerousseau, J., Arnold, G., \& Auvray, M. (personal communication). Visualizing sounds: training-induced plasticity with a visual-to-auditory conversion device. 
Pollok, B., Schnitzler, I., Mierdorf, T., Stoerig, P., \& Schnitzler, A. (2005). Image-to-sound conversion: Experience-induced plasticity in auditory cortex of blindfolded adults. Experimental Brain Research, 167, 287-291.

Proulx, M. J. (2010). Synthetic synaesthesia and sensory substitution. Consciousness and Cognition, 19,501-503.

Proulx, M.J., Brown, D. J., Pasqualotto, A, \& Meijer, P. (2014). Multisensory perceptual learning and sensory substitution. Neuroscience and Biobehavioural Reviews, 41, 16-25.

Proulx, M. J., Gwinnutt, J., Dell'Erba, S., Levy-Tzedek, S., de Sousa, A. A., \& Brown, D. J. (2016). Other ways of seeing: From behavior to neural mechanisms in the online "visual" control of action with sensory substitution. Restorative Neurology and Neuroscience, 34, $29-44$.

Proulx, M. J., Stoerig, P., Ludowig, E., \& Knoll, I. (2008). Seeing 'where' through the ears: effects of learning-by-doing and long-term sensory deprivation on localization based on image-to-sound substitution. PloS one, 3, e1840.

Ptito, M., Iversen, K., Auvray, M., Deroy, O., Kupers, R. (2018). The pluripotent visual cortex in congenital blindness. In F. Macpherson (Ed.). Sensory Substitution and Augmentation, London: Proceedings of the British Academy.

Ptito, M., Moesgaard, S. M., Gjedde, A., \& Kupers, R. (2005). Cross-modal plasticity revealed by electro-tactile stimulation of the tongue in the congenitally blind. Brain, 128, 606-614.

Rieser, J. J., Gum, D. A., \& Hill, E. W. (1986). Sensitivity to perspective structure while walking without vision, Perception, 15, 173-188.

Sekiyama, K. (1991). Importance of head axes in perception of cutaneous patterns drawn on vertical body surfaces. Perception \& Psychophysics, 49, 481-492.

Schinazi, V. R., Thrash, T., \& Chebat, D.-R. (2016). Spatial navigation by congenitally blind individuals. WIREs Cognitive Science, 7, 37-58.

Spence, C. (2011). Crossmodal correspondences: A tutorial review. Attention, Perception \& Psychophysics, 73, 971-995.

Striem-Amit, E., \& Amedi, A. (2014). Visual cortex extrastriate body-selective area activation in congenitally blind people "seeing'" by using sounds. Current Biology, 6, 687-692.

Striem-Amit E, Dehaene S, Cohen L, Amedi A. (2012-a). Reading with sounds: Sensory substitution selectively activates the visual word form area in the blind. Neuron, 3, 640652.

Striem-Amit, E., Guendelman, M., \& Amedi, A. (2012-b). 'Visual' acuity of the congenitally blind using visual-to-auditory sensory substitution. PloS one, 7, e33136.

Striem-Amit, E., Dakwar, O., Reich, L., \& Amedi, A. (2012-c). The large-scale organization of "visual" streams emerges without visual experience. Cerebral Cortex, 22, 1698-1709.

Stroop, J. R. (1935). Studies of interference in serial verbal reactions. Journal of Experimental Psychology, 18, 643-662.

Trivedi, B. (2010). Sensory hijack: rewiring brains to see with sound. Science, 2773, 42-45.

Ward, J, \& Meijer, P. (2010).Visual experiences in the blind induced by an auditory sensory substitution device. Consciousness and Cognition, 19, 492-500.

Ward, J., \& Wright, T. (2014). Sensory substitution as an artificially acquired synaesthesia. Neuroscience and Biobehavioral Review, 41, 26-35.

White, B. W., Saunders, F. A., Scadden, L., Bach-y-Rita, P., \& Collins, C.C. (1970). Seeing with the skin. Perception \& Psychophysics, 7, 23-27. 